\title{
The Capabilities of the Alpha Magnetic Spectrometer as GeV $\gamma$-Rays Detector
}

\author{
R. Battiston ${ }^{1}$ \\ Sezione INFN and Dipartimento di Fisica dell' Università, Perugia, Italia 06100
}

\begin{abstract}
The modeled performance of the Alpha Magnetic Spectrometer (AMS) as a high-energy (0.3 to $100 \mathrm{GeV}$ ) gamma-ray detector is described, and its gamma-ray astrophysics objectives are discussed.
\end{abstract}

\section{INTRODUCTION}

Our knowledge of the $\gamma$-ray sky has increased dramatically during this last decade, due principally to the $\gamma$-ray instruments on board the Compton Gamma Ray Observatory (CGRO) [1]: EGRET, a spark chamber plus calorimeter instrument with $\gamma$-ray sensitivity in the energy interval $30 \mathrm{MeV}$ to $30 \mathrm{GeV}$; COMPTEL,

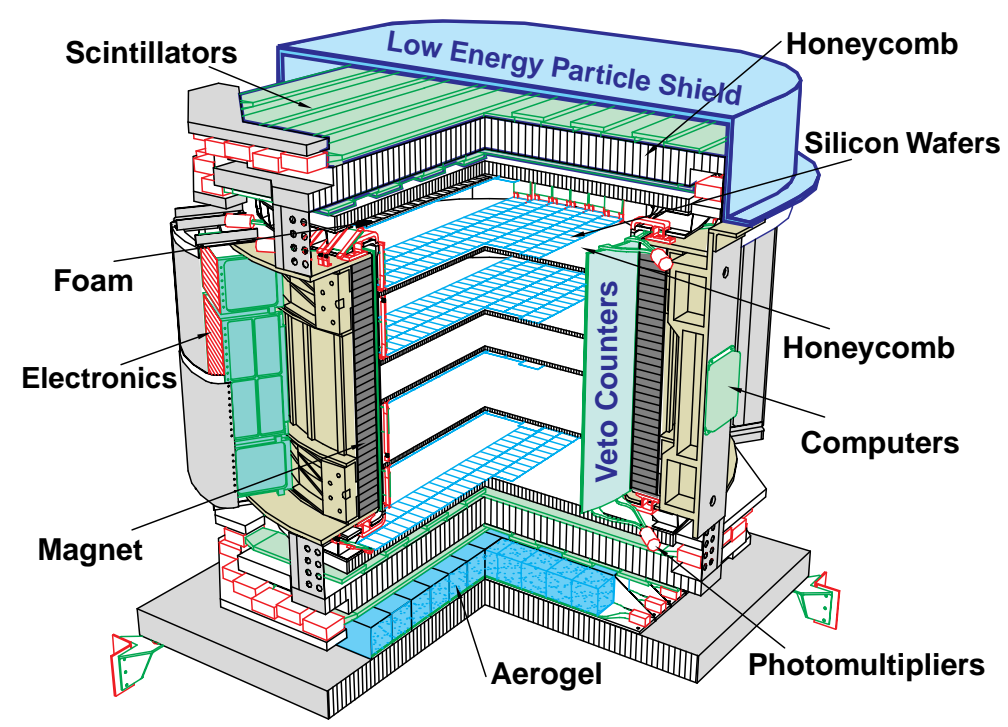

FIGURE 1. A cross section of the baseline AMS instrument.

1) Invited talk at the Workshop on "GeV-TeV Gamma-Ray Astrophysics", 13-16 August 1999, Snowbird, Utah. 
a Compton telescope in the interval 0.1 to $30 \mathrm{MeV}$; BATSE, an omnidirectional x-ray and soft $\gamma$-ray "burst" detector consisting of large NaI scintillators sensitive to $30 \mathrm{keV}$ to $2 \mathrm{MeV}$ photons (with smaller spectroscopic NaI crystals for measurements up to $110 \mathrm{MeV}$ ); and OSSE, consisting of Nai-CsI phoswiches detecting photons of 0.1 to $10 \mathrm{MeV}$. Their observations have revolutionized our understanding of such extragalactic phenomena as blazars and gamma ray bursts (GRBs), as well those within our own Galaxy, such as pulsars.

Until recently, observations with CGRO and other space-based and ground-based telescopes have provided coverage of these and other sources up to the limiting sensitive energy of EGRET, approximately $30 \mathrm{GeV}$. From there, a gap in our knowledge of the $\gamma$-ray sky spectrum has existed up to 200-300 GeV, where ground-based $\gamma$-ray shower detectors presently have their energy thresholds. It is possible that within this gap there are novel features in the $\gamma$-ray sky, such as a gamma-ray line or continuum emission from postulated neutralino annihilation at the center of the Galaxy [22], [23], [24]. Future instruments with sensivity in this unexplored region, such as AGILE [3], recently approved by the Italian Space Agency (ASI), or GLAST [2] being proposed to NASA, may uncover exciting new phenomena.

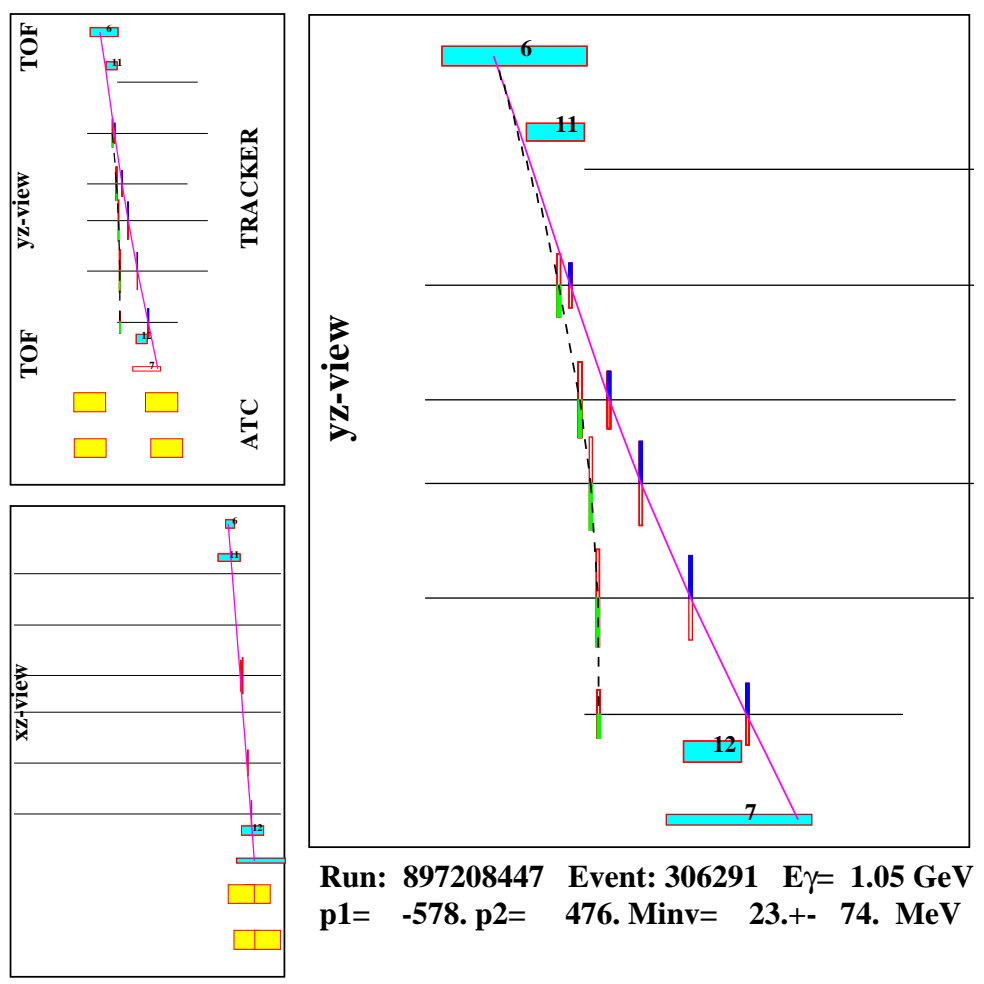

FIGURE 2. A $1 G e V$-ray conversion detected during the STS91 AMS flight.

At the present time, however, our view of the $\gamma$-ray sky has diminished. With the effective turnoff of the EGRET $\gamma$-ray instrument on the CGRO due to the 
nearly complete consumption of its spark chamber gas, there is no operating instrument capable of observing the $\gamma$-ray sky in the energy interval $\sim 10^{-1}$ to $\sim 10^{2}$ GeV. Ground-based $\gamma$-ray detectors, based on the atmospheric Cerenkov technique (ACT) [4], turn on at current energy thresholds of $\sim 200-500 \mathrm{GeV}$. Within the next several years energy thresholds for some ACT observatories are expected to go to as low as 20 to $50 \mathrm{GeV}$, but lower thresholds than these are unlikely to be achieved due to the sizable effect of Earth's magnetic field on the $\gamma$-ray-induced air showers, and the lower Cerenkov photon yield which must be detected against the night sky background. For $\gamma$-ray energies much lower than $10^{2} \mathrm{GeV}$, then, spaced-based detectors are required. This observational gap for the energy window $\sim 10^{-1}$ to $10^{2} \mathrm{GeV}$ will continue to exist for the next several years. Eventually, this gap will be eliminated with the launch of a next-generation $\gamma$-ray satellite, such as GLAST [2], but such a mission is unlikely to occur before the year 2005. Also the AGILE satellite [3], to be launched in 2002, will have a limited sensitivity above $50 \mathrm{GeV}$.

In this paper we describe how the Alpha Magnetic Spectrometer (AMS) can largely fill this gap by acting as a $\gamma$-ray detector with sensitivity in the energy interval of 0.3 to $100 \mathrm{GeV}$ during its three-year mission on board the International Space Station Alpha (ISSA) from 2003 to 2006. AMS, described in detail elsewhere [8], has as primary mission the search for cosmic ray antinuclei as well as the search for dark matter studying anomalies in CR spectra and composition $\left(\right.$ e.g. $\left.e^{+}, \bar{p}\right)$.
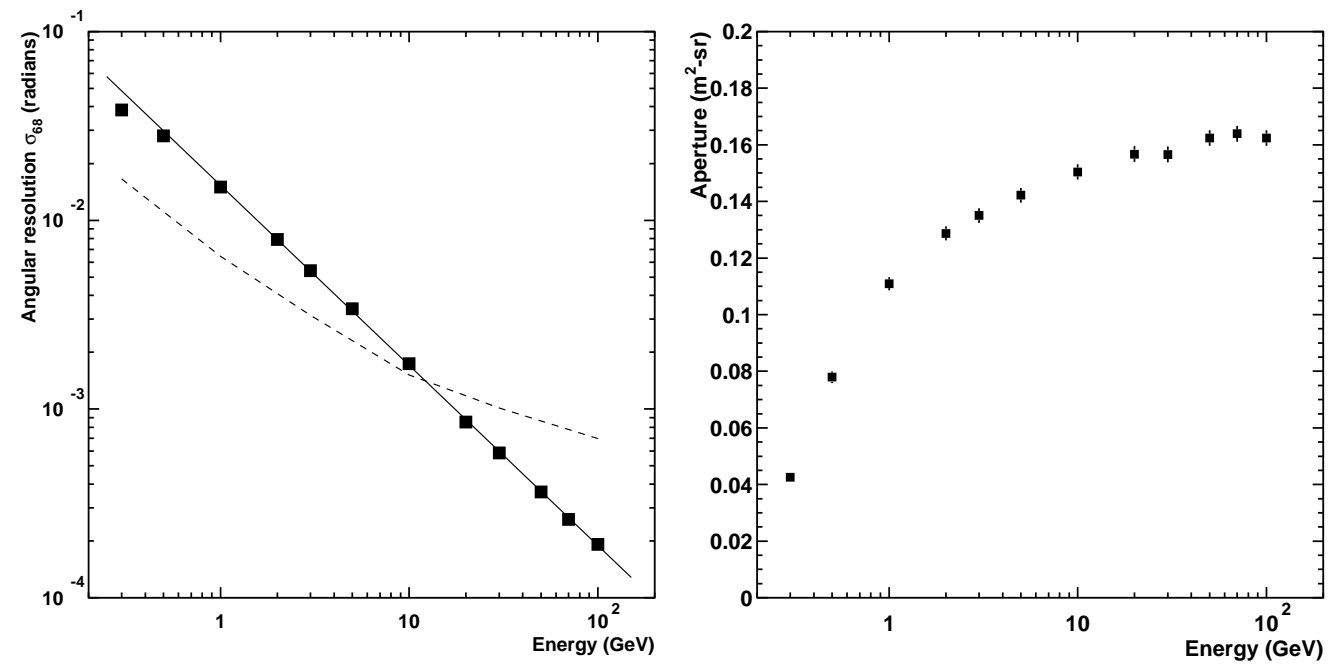

FIGURE 3. (a) Angular resolution of AMS $/ \gamma$ (filled squares) and of GLAST (dashed line) as a function of primary $\gamma$-ray energy in the interval 0.3 to $100 \mathrm{GeV}$;(b) AMS/ $\gamma$ aperture as a function of $\gamma$-ray energy.

There are at least two ways this modification could be done without significantly affecting the experiment sensitivity to antimatter: (a) adding a light (e.g. $0.3 X_{0}$ ) converter at the entrance of the magnetic spectrometer, either passive (e.g., a high-Z 

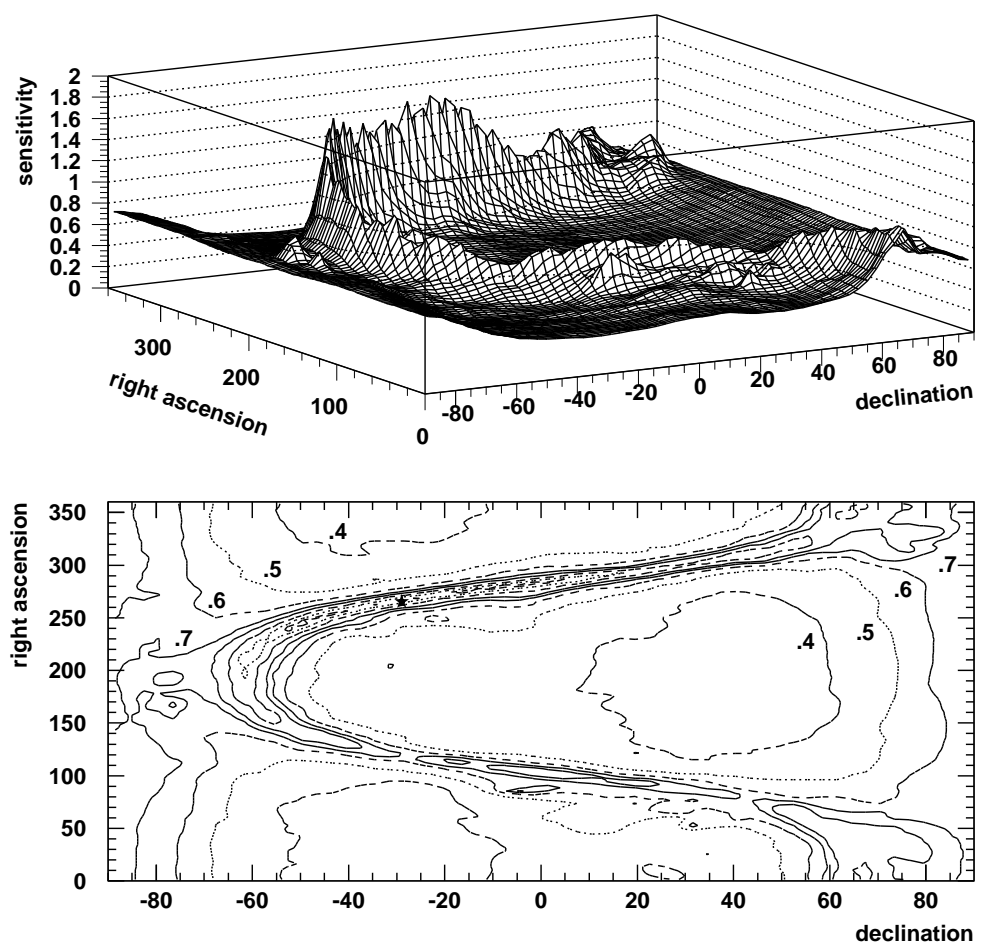

FIGURE 4. A 3-D plot and 2-D contour plot of AMS/ $\gamma$ 's point source sensitivity $n_{0}$ versus celestial coordinates. Recall that $n_{0}$ is the minimum amplitude of the source's differential flux at $1 \mathrm{GeV}$ required for a $5 \sigma$ significance detection. The units for $n_{0}$ in the 3-D plot are $10^{-8} \mathrm{~cm}^{-2} \cdot \mathrm{s}^{-1} \cdot \mathrm{GeV}^{-1}$. Large photon fluxes from the diffuse galactic background are responsible for the deterioration of sensitivity near the Galactic plane.

thin plate) or active (e.g. a multilayer tracking detector), and/or (b) implementing an high granularity imaging shower detector at the bottom of the experiment. In this paper we present a study which has been perfomed on option (a). We will show that in this option, AMS can also detect $\gamma$-rays with performance characteristics similar to EGRET in the energy region of 0.3 to $20 \mathrm{GeV}$, and with significantly enhanced capabilities between 20 and $\sim 100 \mathrm{GeV}$, a region which is not well explored. We refer to this modified instrument as "AMS $/ \gamma$ ". We show that AMS $/ \gamma$ can continue the valuable work of EGRET by providing continued monitoring of extragalactic and galactic $\gamma$-ray sources and by participating in multiwavelength observational campaigns. In addition, AMS $/ \gamma$ will have unprecedented sensitivity to the $\gamma$-ray sky between the energies of 20 to $\sim 100 \mathrm{GeV}$ (albeit at a level somewhat lower than required for detection of known sources with power law spectra), so that $\mathrm{AMS} / \gamma$ might provide us with unexpected discoveries in this region. The next 
section gives a brief description of the baseline AMS instrument and its experience during the first precursor flight. Then we describe the performance characteristics of AMS $/ \gamma$ in option (a) as determined from Monte Carlo analyses and we perform a comparison to EGRET. For a more detailed description of the results of these studies we refer to [5].

\section{THE ALPHA MAGNETIC SPECTROMETER ON THE STS91 FLIGHT}

The Alpha Magnetic Spectrometer has been built by a large international collaboration of high energy physics institutions from the U.S., Italy, China, Finland, France, Germany, Taiwan, Russia, and Switzerland. It recently had a successful test flight on the Space Shuttle mission STS-91 in June 1998 [29], when it was carried in the cargo bay and observed for several days in both the zenith and nadir directions, the latter for measurement of albedo cosmic ray backgrounds. The design of the baseline AMS instrument flown during the STS-91 mission is shown in Figure 1.

The magnet spectrometer consists of a permanent ring dipole magnet made of very high grade $\mathrm{Nd}-\mathrm{B}-\mathrm{Fe}$ rare earth material whose magnetic energy product and residual induction are respectively $(B H)_{\max }>50 \times 10^{6} \mathrm{G}$-Oe and $14,500 \mathrm{G}$, yielding an analyzing power of $\left\langle B L^{2}\right\rangle=0.14 \mathrm{~T}-\mathrm{m}^{2}$ with less than 2 tonnes of magnet mass. Four high precision silicon strip detector tracking planes are located within the magnetic volume, with a fifth and sixth plane located just above and below the magnet [6], [7]. At low rigidities (below $8 \mathrm{GV}$ ) the resolution of the spectrometer is dominated by multiple scattering $(\Delta p / p \sim 7 \%)$, while the maximum detectable rigidity $(\Delta p / p \sim 100 \%)$ is about $500 \mathrm{GV}$. In addition to measuring particle rigidity, the silicon planes will provide six independent measurements of $d E / d x$ for charge determination. Four time-of-flight (ToF) scintillator planes (two above and two below the magnet volume) measure the particle velocity with a resolution of 120 ps over a distance of $1.4 \mathrm{~m}$. The ToF scintillators also measure $d E / d x$, allowing a multiple determination of the absolute value of the particle charge.

A solid state Cerenkov detector below the magnet provides an independent velocity measurement, useful to separate electrons and positrons from the hadronic CR components (protons, helium...) and residual background (pions). In addition, a scintillator anticounter system is located within the inner magnet wall, extending to the ToF scintillators.

The performance of this instrument as a charged cosmic ray detector, and its sensitivity to an antinucleus cosmic ray component, is discussed elsewhere [8].

During the STS-91 AMS has collected about 50 million of single CR events above $\sim 100 \mathrm{MeV} / \mathrm{n}$ kinetic energy, improving the existing cosmic antimatter limits [9] and measuring in details the structure of the CR flux at $400 \mathrm{~km}$ of height and over most of the surface of the earth [10]. 
A few tens of clean two tracks events compatible with $\mathrm{GeV}$-range $\gamma$-ray converting in to an $e^{+} e^{-}$pair on the top layers $(\sim 5 \% X o)$ of the spectrometer have also been observed during the precursor flight (see Figure 2). This is, to our knowledge, the first time high energy $\left.\left(E_{\gamma}>1 \mathrm{GeV}\right)\right) \gamma$-rays are observed in a magnetic spectrometer in space: thanks to the dipolar magnetic field the $e^{+} e^{-}$pair opens up in the bending plane view but it remains very collimated in the non-bending view as it is clearly shown in the Figure. In addition to the particle identification capabilites of the AMS detector, these distinctive topological features of a $\gamma$-ray conversion will be important in rejecting the background induced by the $O\left(10^{5}\right)$ times larger flux of charged CR.

AMS is scheduled to be secured to an external payload attachment point on the International Space Station Alpha in may 2003, where it will remain as a zenithpointing instrument for 3 years of measurement time. We should also note that by the time AMS is attached to the International Space Station it may have undergone significant changes from the baseline design considered here. In particular, the permanent magnet may be replaced by a superconducting magnet, which would considerably improve AMS's $\gamma$-ray detection performance at high energy. Since the detector is still undergoing significant design changes from the baseline instrument flown on the Space Shuttle, we have chosen to fix on that design which currently exists as integrated hardware. The addition of other components discussed in the proposal and currently under developement, such as a transition radiation detector located at the entrance of the magnet and a solid state Čerenkov radiator followed by a segmented calorimeter located at the bottom of the instrument, will improve the particle identification capability of AMS as well as its performance as $\gamma$-ray detector.

\section{THE PERFORMANCE OF AMS $/ \gamma$}

In option (a), the conversion of AMS to $\mathrm{AMS} / \gamma$ requires the addition of two hardware components: (1) A passive or active converter medium, located at the entrance of the spectrometer which converts $\gamma$-rays into electron-positron pairs. (2) A stellar attitude sensor gives the angular orientation of AMS with respect to the celestial sphere to an accuracy of better than $1.5 \times 10^{-4}$ radians.

The determination of the converter thickness is based on an optimization between the probability of $\gamma$-ray convertion, the amount of bremstrahlung losses, which limit the energy resolution, and the amount of multiple scattering, which limits the angular resolution. In addition this modification should not degrade the sensitivity of AMS to the search of antimatter and its particle identification capability. Following the results of our study we choose the value $x=0.3 X_{0}$, for which point source sensitivity is still optimal, the energy resolution is acceptable, and nuclear interaction losses are negligible. For the scope of our study we assumed a $x=0.3 X_{0}$ tungsten plate converter located before the first ToF layer.

A full-instrument GEANT Monte Carlo code was run to determine the per- 
formance of AMS $/ \gamma$. Gamma rays with fixed energies, ranging from 0.3 to 100 $\mathrm{GeV}$, were thrown isotropically at the detector over an opening angle of $50^{\circ}$. All the physical processes for electrons and $\gamma$-rays were "on" in the GEANT code. Bremsstrahlung photons of energies $<20 \mathrm{MeV}$ were not followed, although all bremsstrahlung energy losses were included. Cuts simulating the trigger conditions and the pattern recognition algorithm were applied on the converted events.

Primary $\gamma$-ray energy and incidence direction were determined by adding the fitted momenta vectors of all secondaries evaluated at the converter plate to obtain the primary momentum vector.

Particular care has been given to the study of the instrumental background generated by $\mathrm{CR}$ simulating a $\gamma$-ray conversion. As reference for this study we used the Extragalactic Gamma Ray Background (EGRB) at High Energies. Any measurement of the EGRB requires that instrumental background events be kept to a lower rate than the EGRB flux. To estimate the effects of background due to collisions of cosmic ray electrons, positrons, and protons with the AMS instrument, we divided the $\gamma$-ray spectrum into four bins per decade of energy (from 0.5 to 100 $\mathrm{GeV}$ ) and required that we investigate backgrounds down to a level of $20 \%$ of the EGRB rate in each energy bin. For example, in the $25-40 \mathrm{GeV}$ bin it will take AMS $/ \gamma 2.0 \times 10^{5}$ seconds to obtain 5 EGRB $\gamma$-rays. In our Monte Carlo analysis, therefore, we threw $2.0 \times 10^{5}$ seconds' worth of cosmic ray electron, positron, and proton flux at the instrument to generate a false $\gamma$-ray background. These cosmic rays were thrown isotropically over a zenith angle range of $0^{\circ}<\theta<110^{\circ}$, where the largest zenith angle corresponds to the location of the Earth limb at an orbital altitude of $400 \mathrm{~km}$. The energies were distributed according to known electron [16], [17], positron [18], and proton [19] energy spectra.

Using the same quality cuts to eliminate the electron-induced and proton-induced background events, we had a total of 2 electron-induced and 5 proton induced events; all events had reconstructed energies of $<1 \mathrm{GeV}$, leaving no background events in the interesting high energy region.

\section{Comparison of AMS $/ \gamma$ with EGRET}

Since the basic parameters of AMS $/ \gamma$ are close to those of EGRET in the following we present a comparison among the two detectors. The converter thickness largely dominates all multiple scattering effects, so that the angular and energy resolution of reconstructed primary photons will be completely dominated by multiple Coulomb scattering (MCS) and bremsstrahlung energy losses of the electrons within the converter plate. This is confirmed by the full MC simulation which gives an energy dependence on the angular resolution:

$$
\sigma_{68}^{\mathrm{AMS}}(E)=0.88^{\circ}\left(\frac{E}{1 \mathrm{GeV}}\right)^{-0.956}
$$

which is to be compared to EGRET's angular resolution [12] of 


$$
\sigma_{68}^{\mathrm{EGRET}}(E)=1.71^{\circ}\left(\frac{E}{1 \mathrm{GeV}}\right)^{-0.534} .
$$

In Figure 3a the expected AMS $/ \gamma$ angular resolution is compared to the corresponding figure for GLAST [30], where we note, as it should be expected, that at sufficiently high energy a precise pair spectrometer does eventually give a better angular resolution than a fine grain imaging calorimeter.

An integration of effective area $A(E, \theta)$ over solid angle gives instrument aperture, shown in Figure 3b. Below a $\gamma$-ray energy of $0.3 \mathrm{GeV}$, the converted electrons begin to have too small a radius of curvature to escape from the magnet volume, and detection efficiency plummets. Above $100 \mathrm{GeV}$ the converted electron and positron often do not spatially diverge beyond the two-hit resolution distance of the silicon trackers, causing significant deterioration in $\gamma$-ray energy resolution. These considerations then define the limits of the energy window for AMS $/ \gamma$.

One cannot directly compare the point source sensitivity of AMS $/ \gamma$ to that of EGRET, since AMS is not a pointable instrument. EGRET achieves a flux sensitivity of $I_{\min }(>0.1 \mathrm{GeV}) \approx 10^{-7} \mathrm{~cm}^{-2} \mathrm{~s}^{-1}$ with a 2 -week viewing period. Since it took EGRET one year to map the full sky, where each sky segment was viewed for roughly 2 weeks, we can compare EGRET's sensitivity with that achieved by AMS $/ \gamma$ after one year of operation. By assuming a source differential spectrum of $E^{-2}$, we can convert from EGRET's definition of sensitivity (in terms of integral flux above $0.1 \mathrm{GeV}$ ) to that of $\mathrm{AMS} / \gamma$ (a differential flux above $1 \mathrm{GeV}$ ). In our units the EGRET $5 \sigma$ flux sensitivity is $1 \times 10^{-8}$. Over most of the sky, AMS $/ \gamma^{\prime}$ s mean sensitivity $\left\langle n_{0}\right\rangle$ is estimated to be about a factor of 2 lower than that of EGRET (Figure 4).

The table below summarizes the performance characteristics of AMS as a $\gamma$-ray detector. In particular, by its comparison to EGRET, one sees that the two instruments perform similarly in many respects, one major difference being the energy windows: AMS's energy window is shifted up by roughly one order of magnitude from that of EGRET, thereby providing an improved view of the sky in the region $E_{\gamma} \sim 100 \mathrm{GeV}$.

\begin{tabular}{|l|c|c|}
\hline & AMS & EGRET \\
\hline \hline technique & magn.spectrometer & spark ch.+calorimeter \\
\hline energy window $(\mathrm{GeV})$ & 0.3 to 100. & 0.03 to 30. \\
\hline peak effective area $\left(\mathrm{cm}^{2}\right)$ & 1300 & 1500 \\
\hline angular resolution & $0.77^{\circ}(E / 1 \mathrm{GeV})^{-0.96}$ & $1.71^{\circ}(E / 1 \mathrm{GeV})^{-0.534}$ \\
\hline half-area zenith angle & $\sim 30^{\circ}$ & $\sim 20^{\circ}$ \\
\hline total viewing time $(\mathrm{yr})$ & $\sim 3$ & $\sim 2$ \\
\hline attitude capability & fixed & movable \\
\hline $\begin{array}{l}\text { flux sensitivity } \\
\left(\mathrm{ph} / \mathrm{cm}^{2}{ }_{\text {-S-GeV at } 1 \mathrm{GeV})}\right.\end{array}$ & $\sim 0.5 \times 10^{-8}$ & $\sim 1.0 \times 10^{-8}$ \\
\hline
\end{tabular}

Note that the point source sensitivity is nearly the same for both EGRET and 


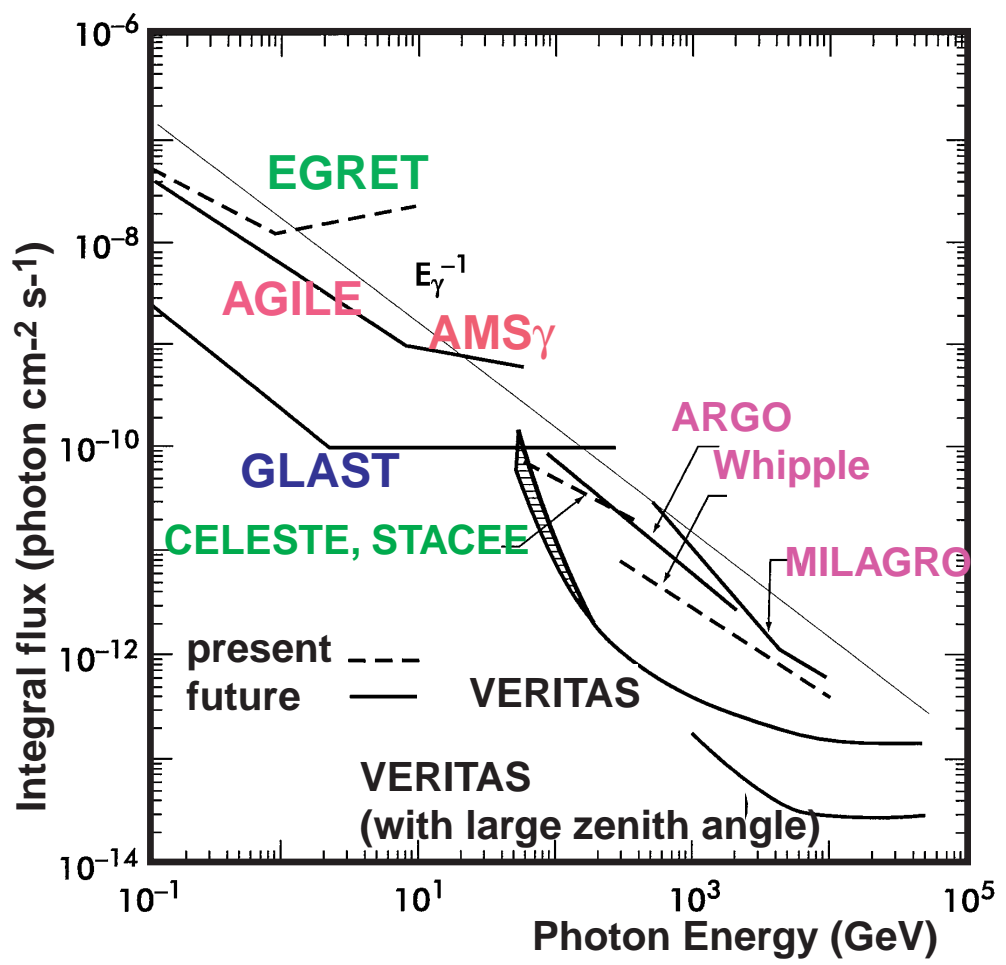

FIGURE 5. Sensitivity of present and future $\gamma$-ray detectors.

AMS, with that of AMS being somewhat (factor $\sim 2$ ) lower. This is because EGRET and AMS have similar effective detection areas and angular resolutions. The fact that AMS's $\gamma$-ray energy threshold is an order of magnitude larger than EGRET's, thereby implying an integrated point source flux about an order of magnitude lower for AMS than for EGRET, is compensated for by the fact that AMS's angular aperture (half-angle area of $\left.30^{\circ}\right)$ is larger than EGRET's $\left(\sim 20^{\circ}\right)$, and by the fact that AMS spends $100 \%$ of its time pointing to the sky (being attached to a gravity-gradient stabilized Space Station), while EGRET typically points one-third of the time to Earth. AMS lacks the low-energy end of EGRET's range due to the curvature of the electron-positron pair in AMS's magnetic field, which limits the detectable $\gamma$-ray energy to $\geq 300 \mathrm{MeV}$. On the other hand, EGRET lacks the high-energy end of AMS's range due to the effect of electromagnetic backsplash in EGRET's NaI calorimeter which vetos most events above $30 \mathrm{GeV}$. 
Since AMS $/ \gamma$ and EGRET have very similar $\gamma$-ray detection capabilities, AMS $/ \gamma$ will be able to continue and extend the investigation of galactic and extragalactic $\gamma$-ray sources initiated by EGRET. One main difference between the two detectors, that of significant aperture above $30 \mathrm{GeV}$ for $\mathrm{AMS} / \gamma$, may lead to the observation of new phenomena in this relatively uncharted region.

Figure 5 shows a comparison between the sensitivity of present and future high energy $\gamma$-ray detectors.

\section{CONCLUSIONS}

We have shown that with minor modifications the Alpha Magnetic Spectrometer can become a powerful $\gamma$-ray detector as well, with overall performance characteristics being comparable, if not superior, to those of EGRET. With $\gamma$-ray energy resolution extending past $100 \mathrm{GeV}$, and with an aperture that is nearly flat above $\sim 3 \mathrm{GeV}, \mathrm{AMS} / \gamma$ can address a number of outstanding issues in $\gamma$-ray astrophysics that relate to the relatively unexplored region of $E_{\gamma}=20-200 \mathrm{GeV}$. For one, AMS $/ \gamma$ will likely confirm or refute the hypothesis that unresolved blazars are responsible for the bulk of the extragalactic $\gamma$-ray background; AMS $/ \gamma$ will also extend the spectrum of the diffuse galactic background to above $100 \mathrm{GeV}$, helping to resolve current difficulties in interpreting the EGRET diffuse galactic background measurement [28].

AMS $/ \gamma$ should roughly double the total number of blazars detected in $\gamma$-rays, and will be enable multiwavelength observational campaigns to include the $\mathrm{GeV}$ region of blazar spectra during the flight years of 2003-2006. There is an additional possibility that an indirect detection of the cosmic UV and optical photon background can be made through the detection of extinctions in high-redshift blazars above $\sim 20 \mathrm{GeV}$. AMS $/ \gamma$ will also likely observe $\mathrm{GeV} \gamma$-ray emission from one or more gamma ray bursts during its operational lifetime.

AMS $/ \gamma$ will also search for both line and continuum emission of $\gamma$-rays from the region of the Galactic Center created by the annihilation of dark matter neutralinos. Although the sensitivity to line emission appears marginal, there is nevertheless a finite, though small, region of halo/MSSM phase space which allows a detection by

AMS. However, a much larger region of dark-matter halo/MSSM parameter space can be constrained in a search for continuum $\gamma$-rays by $\mathrm{AMS} / \gamma$.

Finally, we note that even higher sensitivities can be reached with the addition of a high granularity calorimeter below the magnet (option (b)); this will be the subject of future work.

\section{REFERENCES}

1. J.D. Kurfess, D.L. Bertsch, G.J. Fishman, and V. Schöfelder (1997) AIP Conf. Proc. 410,509 .

2. D. Engovatov et al. (1997) IEEE Nucl. Sci. Symp. (Albuquerque). 
3. AGILE, Phase A Report, ASI Small Program Scientific Mission, October 1998, AR.DAS.98.501.

4. A.M. Hillas and J.R. Patterson (1990) J. Phys. G Nucl. Part. Phys. 16, 1271.

5. R. Battiston, M. Biasini, E. Fiandrini, J. Petrakis and M.H. Salamon, to be published on Astropart. Phys., astro-ph/9909432.

6. M. Acciarri et al. (1995) Nucl. Instr. Meth. A360, 103; R. Battiston (1995) Nucl. Phys. B Proc. Suppl. 44, 274.

7. W. Burger, in Proceedings of Vertex 1999.

8. S. Ahlen et al. (1994) Nucl. Instr. Meth. A350, 351.

9. AMS Collaboration, (1999) Phys. Lett. B461, 387.

10. U. Becker, invited talk at 26th ICRC (1999), (Salt Lake City); AMS Collaboration submitted to Phys. Lett. B.

11. R. Mukherjee, et al. (1997) Ap.J. 490, 116.

12. D.J. Thompson, et al. (1993) Ap.J.Suppl. 86, 629.

13. J.R. Mattox, et al. (1997) Ap.J. 481, 95.

14. R.C. Hartman, W. Collmar, C. von Montigny, and C.D. Dermer (1997) AIP Conf. Proc. 410, 307.

15. D.J. Thompson, et al. (1995) Ap.J.Suppl. 101, 259.

16. D. Müller and K.K. Tang (1987) Ap.J. 312, 183.

17. R.L. Golden, et al. (1994) Ap.J. 436, 769.

18. S.W. Barwick, et al. (1997) Ap.J. 482, L191.

19. W. Menn, et al. (1997) 25th ICRC 3, 409 (Dublin).

20. G. Alverson, et al. (1993) Phys. Rev. D48, 5.

21. M. Mori (1997) Ap.J. 478, 225.

22. G. Jungman, M. Kamionkowski, and K. Griest (1996) Phys. Rep. 267, 195.

23. P. Ullio and L. Bergström (1997) hep-ph/9707333.

24. L. Bergström and P. Ullio (1997) hep-ph/9706232.

25. L. Bergström, P. Ullio, and J.H. Buckley (1997) astro-ph/9712318.

26. T. Sjöstrand (1994) Comp. Phys. Comm. 82, 74.

27. M. Biasini (1999) in Proc. XIII Rencontres de Physique (La Thuile).

28. M. Pohl and J.A. Esposito (1998) Ap. J. 507, 327.

29. R. Battiston (1999) in Proc. XIII Rencontres de Physique (La Thuile); also as astro$\mathrm{ph} / 9907152$.

30. E.D. Bloom (1996) Sp. Sci. Rev. 75, 109. 